Rodríguez-Pleguezuelo et al. Terreno y vegetación autóctona vs. agricultura Ecosist. Recur. Agropec. 4(12):499-509,2017

\title{
La fruticultura subtropical en los ecosistemas de ladera de clima mediterráneo: cambios en la vegetación
}

\section{Subtropical fruit farming in Mediterranean weather hillside ecosystem: changes in vegetation}

\author{
Carmen Rocío Rodríguez-Pleguezuelo ${ }^{1}$, José Ramón Francia-Martínez ${ }^{2}$, Dionisio Franco-Tarifa ${ }^{3}$, Víctor \\ Hugo Durán-Zuazo ${ }^{4 *}$ \\ ${ }^{1}$ Earth and Life Institute. Environmental Sciences. UniversitéCatholique de Louvain. Croix du Sud 2, bte L7.05.02. B-1348 \\ Louvain-la-Neuve, Belgium \\ ${ }^{2}$ IFAPA Centro "Camino de Purchil". Apdo. 2027, 18080. Granada, España \\ ${ }^{3}$ Finca Experimental "El Zahori", Ayuntamiento de Almuñécar, Plaza de la Constitución 1, Almuñécar, Granada \\ ${ }^{4}$ IFAPA Centro Las Torres-Tomejil, Crtra Sevilla-Cazalla, km 12.2, 41200 Alcalá del Rio, Sevilla, España \\ ${ }^{*}$ Autor de correspondencia: victorh.duran@juntadeandalucia.es
}

Artículo científico recibido: 04 de mayo de 2016 aceptado: 01 de febrero de 2017

RESUMEN. En la costa de Granada, sureste de España, se encuentra una zona de frutales subtropicales en laderas, distribuidos en terrazas. Se evaluaron tasas de descomposición y liberación de nitrógeno $(\mathrm{N})$ de hojarasca, en dos ecosistemas: (1) ecosistema de ladera inalterado (ELI) sin cultivo agrícola, con cubierta de vegetación autóctona, y (2) ecosistema de ladera alterado (ELA) con frutales de mango (Mangifera indica L.), aguacate (Persea americana Mill.), chirimoyo (Annona cherimolia Mill.), níspero (Eriobotrya japonica (Thunb.) Lindl.), y cubiertas de plantas aromáticas y medicinales (PAM) en los taludes de las terrazas. En ELA la tasa de descomposición de hojarasca de chirimoyo fue la más alta, y entre las PAM la alhucema rizada (Lavandula dentata L.), así como la mejorana (Thymus mastichina L.), alhucema rizada (Lavandula dentata L.) y ajedrea (Satureja obovata Lag.) registraron altas tasas de liberación de N. En ELI acebuche (Olea europea L.) y bolina (Genista umbellata (L'Hér.) Dum., tuvieron bajas tasas de descomposición. Las PAM en ELA pueden contribuir a restablecer los ciclos naturales de reciclado de nutrientes, minimizando los efectos del cambio de uso del suelo.

Palabras clave: Ciclo del nitrógeno, cultivos subtropicales, descomposición de hojarasca, terrazas de cultivo.

ABSTRACT. The coast of Granada, in southeast Spain, has a zone of subtropical fruits in hillsides, distributed in terraces. Nitrogen $(\mathrm{N})$ breakdown and release rates for litter were assessed in two ecosystems: (1) unaltered hillside ecosystem (ELI) without agricultural crops, with indigenous vegetation cover and (2) altered hillside ecosystem (ELA) with mango (Mangifera indica L.), avocado (Persea americana Mill.), cherimoya (Annona cherimolia Mill.), loquat (Eriobotrya japonica (Thunb.) Lindl.) and covered with aromatic and medicinal plants (PAM) in terrace slopes. In the ELA, the cherimoya litter breakdown rate was higher; among the PAM, fringed lavender (Lavandula dentata L.), mastic thyme (Thymus mastichina L.) and savory (Satureja obovata Lag.) recorded high Nitrogen release rates. In ELI, olive (Olea europea L.) and bolina (Genista umbellata (L'Hér.) Dum., had low breakdown rates. The PAM and ELA may contribute to resetting natural nutrient recycling cycles, minimizing the effects of change in land use.

Key words: Nitrogen cycle, subtropical crops, litter breakdown, cropping terraces.

\section{INTRODUCCIÓN}

La descomposición de la hojarasca es el conjunto de procesos físicos y químicos relacionados con la transformación de la misma hasta sus constituyentes químicos elementales. Es un proceso determinante en los ciclos de nutrientes de la mayor parte de los ecosistemas terrestres (Castellanos y León 


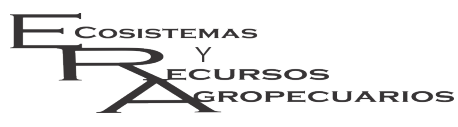

Rodríguez-Pleguezuelo et al.

Terreno y vegetación autóctona vs. agricultura

Ecosist. Recur. Agropec.

4(12):499-509,2017

2011). En este sentido, la liberación de nutrientes de la hojarasca es un factor en la productividad primaria de los ecosistemas, ya que estos nutrientes vuelven a estar disponibles para las plantas (SantaRegina et al. 1997), jugando un papel fundamental en el flujo de carbono (Sun et al. 2004).

De forma general, la tasa de descomposición de la hojarasca está controlada por condiciones climáticas como la temperatura media, la precipitación y las tasas de evapotranspiración (O’Neill et al. 2003, Zhang et al. 2008), la composición química del material y por los organismos edáficos (Wang et al. 2015). El clima influye de forma directa en la descomposición de la hojarasca por la temperatura y la humedad, de forma indirecta por la influencia en la composición de las comunidades vegetales, determinando el tipo de material a descomponer (Aerts 1997). En términos del tipo de hojarasca, se pueden distinguir tres fracciones principales; la primera es la fracción fácilmente soluble, que desaparece de forma rápida, la segunda es la fracción no soluble, de forma fácilmente degradable, compuesta por celulosa y hemicelulosa; y la tercera permanece mucho más tiempo de forma inalterada, se compone de lípidos, ligninas y carbohidratos lignificados (Heal et al. 1997). La relación entre el tipo de hojarasca y las tasas de descomposición ha sido demostrada por Kögel-Knabner (2002), Sariyildiz y Anderson (2003) y Zhou et al. (2008).

$L a$ a relación $C: N$ es un buen índice de la susceptibilidad de la degradación de la hojarasca. Hojarascas con relaciones bajas de C:N se descomponen de forma más rápida que aquellas con relaciones mayores (Taylor et al. 1989). La descomposición de la hojarasca es un proceso que se ha estudiado para ecosistemas tropicales y subtropicales (Pandey et al. 2007), semiáridos (Tateno et al. 2007), templados (Cookson et al. 2007) y mediterráneos (Sirulnik et al. 2007). Pero en ecosistemas forestales con sistemas de cultivo, se han realizado pocos estudios (Romeo et al. 2012)

En la región mediterránea, y sobre todo en la semiárida del sureste de España, la degradación del suelo es un problema de importancia, debido a las acciones de tipo antrópico (Durán et al. 2005,
2013) y a los largos periodos de sequía seguidos de lluvias intensas e irregulares (Moriondo et al. 2006, Rodríguez-Díaz et al. 2007). Una de las causas de la erosión del suelo es la eliminación de la vegetación natural (Durán et al. 2014). En este contexto, los arbustos, plantas representativas de los ecosistemas mediterráneos degradados reciben atención particular, ya que son esenciales para controlar la erosión de los suelos (Durán y Rodríguez 2009, Durán et al. 2011). Por otra parte, la perturbación del terreno en laderas para la construcción de terrazas e implantación de la agricultura, es una práctica muy extendida en la costa de Granada (Durán et al. 2013), lo cual conlleva una serie de efectos negativos en el medio ambiente (Durán et al. 2005, Rodríguez et al. 2011). Se distinguen dos escenarios de estudio, el ecosistema de ladera inalterado (ELI) y el ecosistema de ladera alterada (ELA). Este último consiste de especies frutales tropicales y subtropicales en terrazas de cultivo, con medidas de control de erosión con plantas aromáticas y medicinales en los taludes de estas terrazas. Mientras que en ELI prevalece una mezcla de plantas herbáceas anuales y bianuales combinadas con diferentes arbustos leñosos espontáneos y típicos de ambientes. Por lo anterior, el objetivo del estudio fue analizar la descomposición de la hojarasca y el reciclaje de nitrógeno generado por la introducción de cultivos tropicales y subtropicales en ladera.

\section{MATERIALES Y MÉTODOS}

\section{Zona de estudio}

Este estudio se llevó a cabo en la finca experimental El Zahorí en Almuñécar, Granada, España, ubicada a los $36^{\circ} 48^{\prime} 00^{\prime \prime} \mathrm{LN}$ y $3^{\circ} 38^{\prime} 00^{\prime \prime}$ LO a $180 \mathrm{msnm}$. El clima de la zona es Mediterráneo subtropical, con una temperatura media anual de $20.8{ }^{\circ} \mathrm{C}$ y precipitación promedio de $449.0 \mathrm{~mm}$ (Elías y Ruiz 1977). Los suelos son por general Xerorthent Típicos y Regosoles Eútricos, con $684 \mathrm{~g}$ $\mathrm{kg}^{-1}$ de arena, $235 \mathrm{~g} \mathrm{~kg}^{-1}$ de limo, $81 \mathrm{~g} \mathrm{~kg}^{-1}$ de arcilla, $5.4 \mathrm{~g} \mathrm{~kg}^{-1}$ de carbono orgánico, así como $0.7 \mathrm{~g} \mathrm{~kg}^{-1}$ de N, $14.6 \mathrm{mg} \mathrm{kg}^{-1}$ de P y $178.7 \mathrm{mg}$ $\mathrm{kg}^{-1}$ de K asimilable (FAO 1998, Soil Survey Staff 


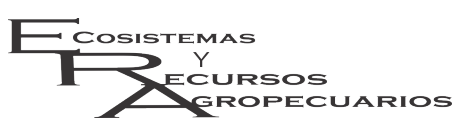

Rodríguez-Pleguezuelo et al.

Terreno y vegetación autóctona vs. agricultura

Ecosist. Recur. Agropec.

4(12):499-509,2017

1999). Las terrazas de estudio por lo general se establecen en hileras simples de árboles frutales, las cuales miden de 2 a $3 \mathrm{~m}$ de ancho, con talud entre 3 y $5 \mathrm{~m}$, y longitud de 160 a $170 \mathrm{~m}$.

\section{Colecta de hojarasca}

Se utilizó la técnica de las bolsas de red de nailon, debido a que es el método estandarizado para la colecta de hojarasca y la evolución de su descomposición en este tipo de estudios (Aerts 1997). El escenario inalterado (ELI), consistió en vegetación espontánea arbustiva de la zona, para lo cual se emplearon parcelas de muestreo de $25 \mathrm{~m}^{2}$, superficie mínima recomendada para colectar hojarasca en arbusto (Barkman 1989). En estas laderas inalteradas, la mezcla de plantas herbáceas anuales y bianuales espontaneas (PHAs) se colectaron de forma aleatoria en cuadrantes de $50 \times 50 \mathrm{~cm}$, siendo las plantas más comunes amapola silvestre ( $\mathrm{Pa}$ paver rhoeas L.), correhuela (Convolvulus arvensis L.), reseda (Reseda phyteuma L.), manzanilla silvestre (Anacyclus sp.), mostaza silvestre (Sinapis arvensis L.), alfalfa (Medicago sp.), poa anual (Poa annua L.), y malva común (Malva sylvestris L.) Mill.). Las especies más representativas de arbustos fueron retama (Retama sphaerocarpa L.), espliego (Lavanadula officinalis Chaix), bolina (Genista umbellata (L'Hér.) Dum.), acebuche (Olea europea L. cv. sylvestris) y matagallo (Phlomis purpurea L.).

El escenario alterado (ELA) fue una zona de frutales subtropicales con cubiertas vegetales diversas, usadas para proteger el suelo de la erosionen (Figura 1). En ELA, las hojas de los frutales cultivados de aguacate (Persea americana Mill.), chirimoyo (Annona cherimolia Mill.), níspero (Eriobotrya japonica Lindl.) y mango (Mangifera indica L). se recogieron en bolsas de $50 \times 25 \mathrm{~cm} \times 1 \mathrm{~mm}$ de luz de la zona media del árbol, de acuerdo con los puntos cardinales y de las ramas de edad fisiológica similar, al mismo tiempo se recolectaron en bolsas de $24 \times 15 \mathrm{~cm} \times 1 \mathrm{~mm}$ de luz las plantas aromáticas y medicinales (PAM) de mejorana ( $T$. mastichina L), lavanda (L. dentata L.), ajedrea (Satureja obovata Lag.), albaida (Anthyllis cytisoides L.), y espliego ( $R$. officinalis L. cv. Postratum) empleadas para proteger el suelo de la erosión en los taludes (Figura 2). El tamaño de apertura de la malla fue de $1 \mathrm{~mm}$, lo suficientemente pequeño para impedir pérdidas importantes de material, y para permitir la actividad microbiana aeróbica y la entrada de pequeños animales del suelo que se excluyeron de la descomposición (Dutta y Agraval 2001).

La descomposición de la hojarasca se realizó en bolsas de nailon por 12 meses. Para cada tipo de plantas, se enterraron entre 24 y 36 bolsas a profundidad de entre 10 y $15 \mathrm{~cm}$, las cuales se recuperaron cada dos meses. En cada recogida, se extrajeron entre 6 y 10 bolsas para cada tipo de vegetación (Tabla 1).

\section{Determinaciones y análisis estadístico}

A las bolsas recuperadas del suelo, se les eliminó el suelo adherido, los restos de otras plantas y material exógeno. Para luego lavarlas con agua corriente y después con agua destilada. Posteriormente se secaron en estufa a $70{ }^{\circ} \mathrm{C}$ hasta peso constante y pesaron para determinar la biomasa residual. La pérdida de masa en el tiempo se expresó con el modelo de descomposición exponencial $M_{t}=M_{0} e^{-k t}$. Donde: $\mathrm{Mt}=$ masa en el instante $t$, Mo: masa al inicio y $t=$ el tiempo. Para luego calcular las constantes de descomposición $\left(\right.$ años $\left.^{-1}\right)$ (Olson 1963): $k=-\ln \left(M_{t} / M_{0}\right)$. El tiempo medio de residencia de $\left(R_{t}\right)$ de la hojarasca de cada tipo de planta se estimó por medio del inverso de $k$ (Waring y Schlesinger 1985): $R_{t}=1 / k$. Para determinar el carbono (C) y nitrógeno $(N)$ en la biomasa inicial, así como en la remanente de las bolsas, se utilizó un analizador elemental Fisons Carlo Erba EA 1108 CHNS-O.

La biomasa restante en cada recogida, los tiempos de permanencia, el contenido de $\mathrm{C}$ y N, y la relación $\mathrm{C}: \mathrm{N}$ se evaluó con análisis de varianza, con el tiempo y las especies como factores principales, con el paquete estadístico SPSS 15.0. EI porcentaje de elementos remanente $(\mathrm{C}$ y $N)$ se calculó como la proporción entre el contenido en el instante $T_{i}$ y su contenido inicial $\left(T_{0}\right)$. 
Rodríguez-Pleguezuelo et al.

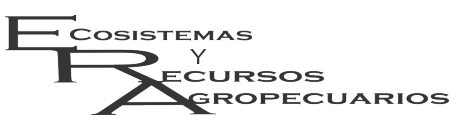

Terreno y vegetación autóctona vs. agricultura

Ecosist. Recur. Agropec. 4(12):499-509,2017

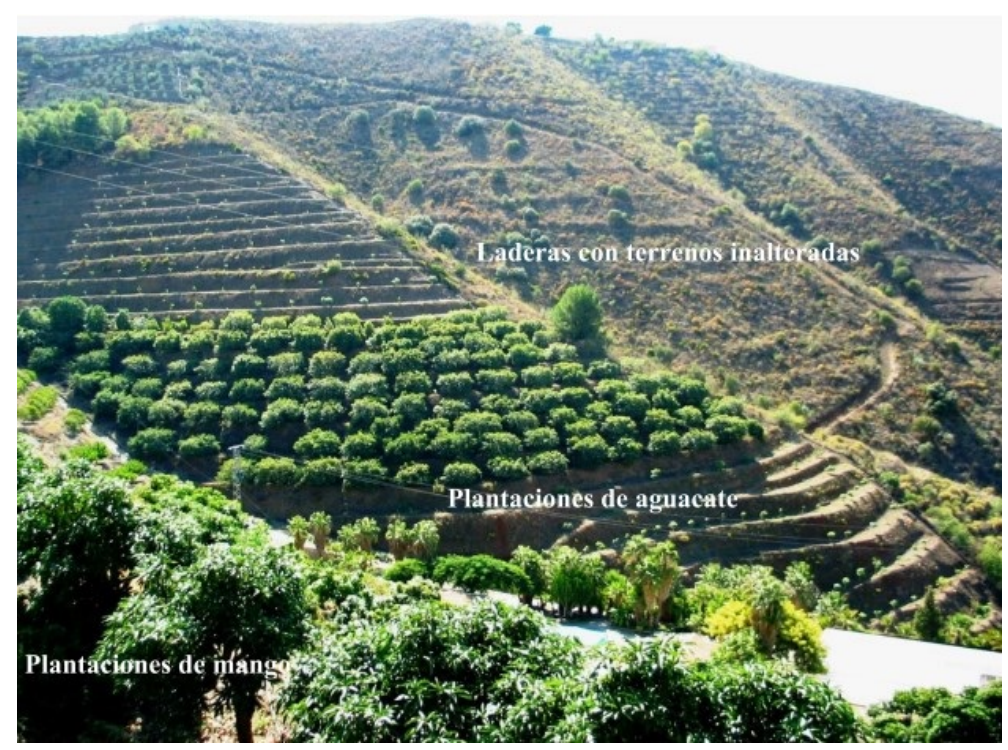

Figura 1. Laderas alteradas por la construcción de terrazas de cultivo y laderas inalteradas con vegetación espontánea.

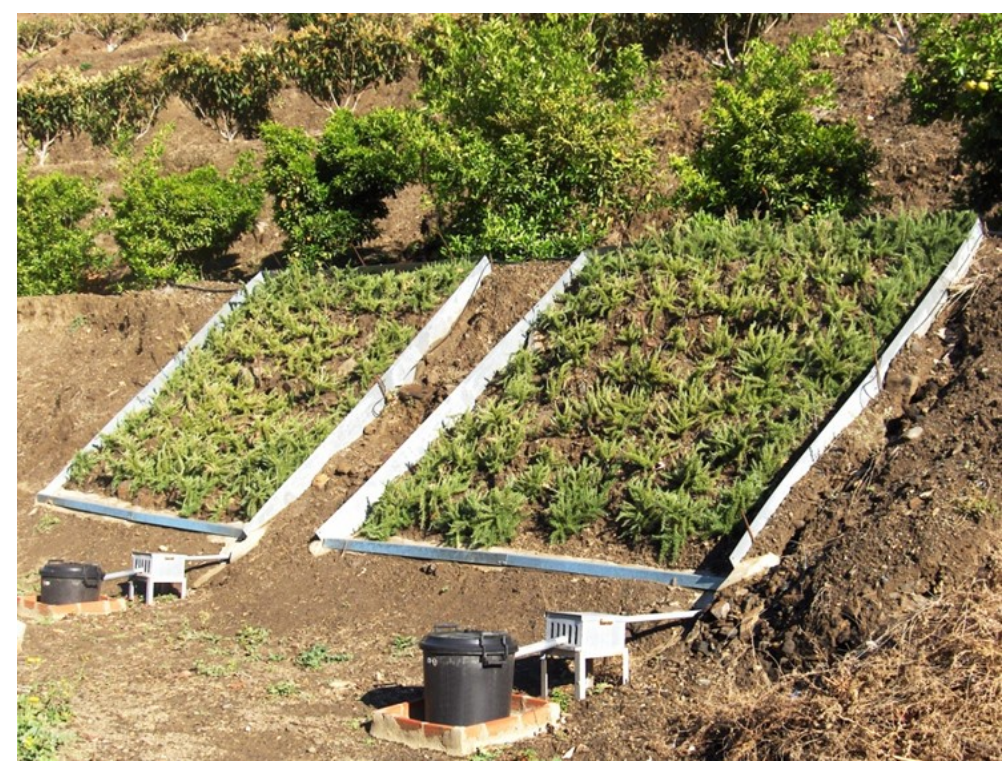

Figura 2. Plantas aromáticas y medicinales empleadas para el control de la erosión de suelos en los taludes de las terrazas de cultivo.

\section{RESULTADOS}

La Figura 3a describe la dinámica de la pérdida de masa de la hojarasca mediante un modelo de descomposición exponencial simple. El valor de $k$ alcanzó su máximo para chirimoyo a los 1.30 años $^{-1}$ y el mínimo para mango a los 0.64 años $^{-1}$. A los 159 $\mathrm{d}$ la biomasa restante en mango, níspero, aguacate y chirimoyo fue de $64.7,60.6,54.5$ y $37.6 \%$, respectivamente. Lo que demuestra que la descomposición de la masa foliar fue función de las particularidades de cada especie. Los tiempos de residencia $\left(R_{t}\right)$ 
Tabla 1. Tiempo de recolecta y tipos de vegetación estudiados

\begin{tabular}{|c|c|c|c|c|c|}
\hline Vegetación & $\begin{array}{l}\text { Tiempo } \\
\text { (meses) }\end{array}$ & $\begin{array}{l}\text { Tiempo recolecta de } \\
\text { la muestra (meses) }\end{array}$ & $\begin{array}{l}\text { Número } \\
\text { recolectas }\end{array}$ & $\begin{array}{l}\text { Número bolsa } \\
\text { por recogida }\end{array}$ & $\begin{array}{c}\text { Total de } \\
\text { bolsas }\end{array}$ \\
\hline \multicolumn{6}{|l|}{ Ecosistema de ladera inalterado (ELI) } \\
\hline PHAs & 12 & 3 & 4 & 6 & 24 \\
\hline $\begin{array}{l}\mathrm{RS}, \mathrm{LO}, \mathrm{GU}, \mathrm{OE}, \mathrm{PP} \\
\text { Ecosistema de ladera alterado (ELA) }\end{array}$ & 12 & 2 & 6 & 6 & 36 \\
\hline $\mathrm{SO}, \mathrm{AC}, \mathrm{RO}$ & 14 & 2 & 4 & 8 & 32 \\
\hline PA, MI, ACh, EJ & 18 & 2 & 3 & 8 & 24 \\
\hline TM, LD & 12 & 3 & 4 & 6 & 24 \\
\hline
\end{tabular}

fueron para chirimoyo, níspero, aguacate y mango de $0.77,1.05,1.11$ y 1.56 años, respectivamente (Figura 4). La biomasa remanente al final del ensayo (365 d) y la relación C:N están relacionadas de forma significativa $\left(R^{2}=0.70\right)$.

En relación a las plantas aromáticas y medicinales, las constantes de descomposición $k$ para T. mastichina L., R. officinalis L., A. cystisoides L., S. obovata Lag y L. dentata L. fue de $1.40,1.06,1.30,1.25$ y 1.87 años $^{-1}$, respectivamente (Figura 3 y 4 ). Al final del ensayo, la biomasa remanente fue de $29.0,27.7,21.5,19.7$, y $17.7 \%$, respectivamente. Comparando entre si las PAM estudiadas, el porcentaje de biomasa remanente para L. dentata L. y S. obovata Lag. fue un 39 y 32 $\%$ menor que para Thymus mastichina L., que alcanzó el mayor porcentaje de biomasa remanente. Mientras que $R$. officinalis $\mathrm{L}$. cv postratum y $T$. mastichina $\mathrm{L}$. fueron las más persistentes con menor tasa de descomposición. La biomasa remanente al final del experimento (BRf) y la relación C: $\mathrm{N}$ estuvieron relacionadas de forma significativa (BRf $=$ $0.30 \mathrm{C}: \mathrm{N}+12.3, \mathrm{R} 2=0.96$ ). Es importante señalar que cuando $T$. mastichina L. se incluyó en el cálculo de la regresión, la relación no fue significativa, ya que esta planta tuvo una velocidad de descomposición lenta con mayor relación $\mathrm{C}: \mathrm{N}$.

El material vegetal de $L$. officinalis Chais, $P$. purpurea L., R. sphaerocarpa L., O. europaea L. y G. umbellata (L'Hér.) Dum., alcanzó tasas de descomposición de hojarasca de 1.06, 1.74, 11.71, 1.18 y 1.01 años $^{-1}$, respectivamente (Figura 3b y 4). Para la mezcla de plantas anuales y bianuales, se calculó una tasa de 3.23 años $^{-1}$. Como cabe esperar, que este tipo de plantas alcanzan las tasas más altas, que fueron entre 2.7 y 3.2 veces mayores que las de los arbustos de G. umbellata (L'Hér.) Poir. y O. europea $L$. Después de un año $L$. officinalis Chais y $P$. purpurea L. perdieron el 85 y $80 \%$ de su masa inicial, mientras que G. umbellata (L'Hér.) Dum.y $O$. europaea L. perdieron el 37.9 y $32.6 \%$ de su masa, respectivamente. En los arbustos estudiados se observó una fase inicial caracterizada por una pérdida rápida de biomasa, que fue más pronunciada en $L$. officinalis y $R$. sphaerocarpa L., para las cuales se registraron descenso de biomasa de más del $50 \%$ con respecto a la inicial. Para los cinco arbustos, la relación $\mathrm{C}: \mathrm{N}$ fue un buen índice de la biomasa remanente al final del experimento $\left(R^{2}=0.93\right)$.

\section{Dinámica global del nitrógeno en los ecosis- temas estudiados}

En ambos agroecosistemas, se observa la inmovilización del N (Tabla 2). Los valores negativos indican que la concentración de $\mathrm{N}$ en la hojarasca restante aumentó con respecto a la concentración inicial, por lo que hubo una inmovilización del $\mathrm{N}$. Los valores positivos implican, que la concentración en la biomasa remanente disminuyó con respecto a la inicial, ocurriendo la liberación de N. El sistema de ecosistema de ladera alterado, presentó valores medios de 1.15 y 0.20 , respectivamente; con valor medio de 0.68. Sin embargo, el valor de $\mathrm{N}$ para el ecosistema de ladera inalterado fue 2.4 veces mayor que para ELA, con valor global medio de 1.64 (Tabla 3). 
Rodríguez-Pleguezuelo et al.
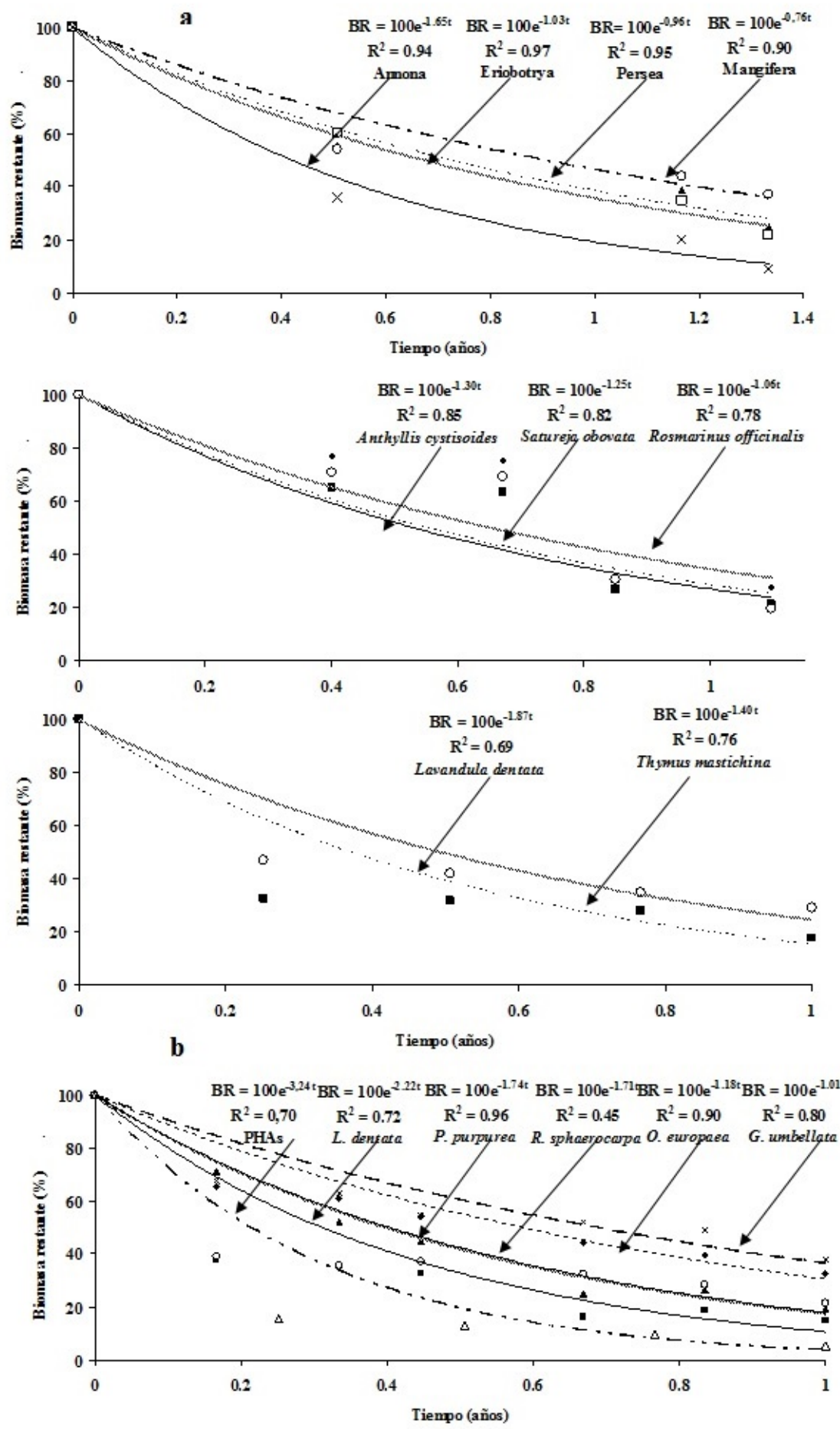

Figura 3. Modelo exponencial para expresar el porcentaje de biomasa restante con el tiempo para los escenarios ecosistema de ladera alterado (a) y ecosistema de ladera inalterado (b). BR: biomasa restante, PHA: plantas herbáceas anuales. 


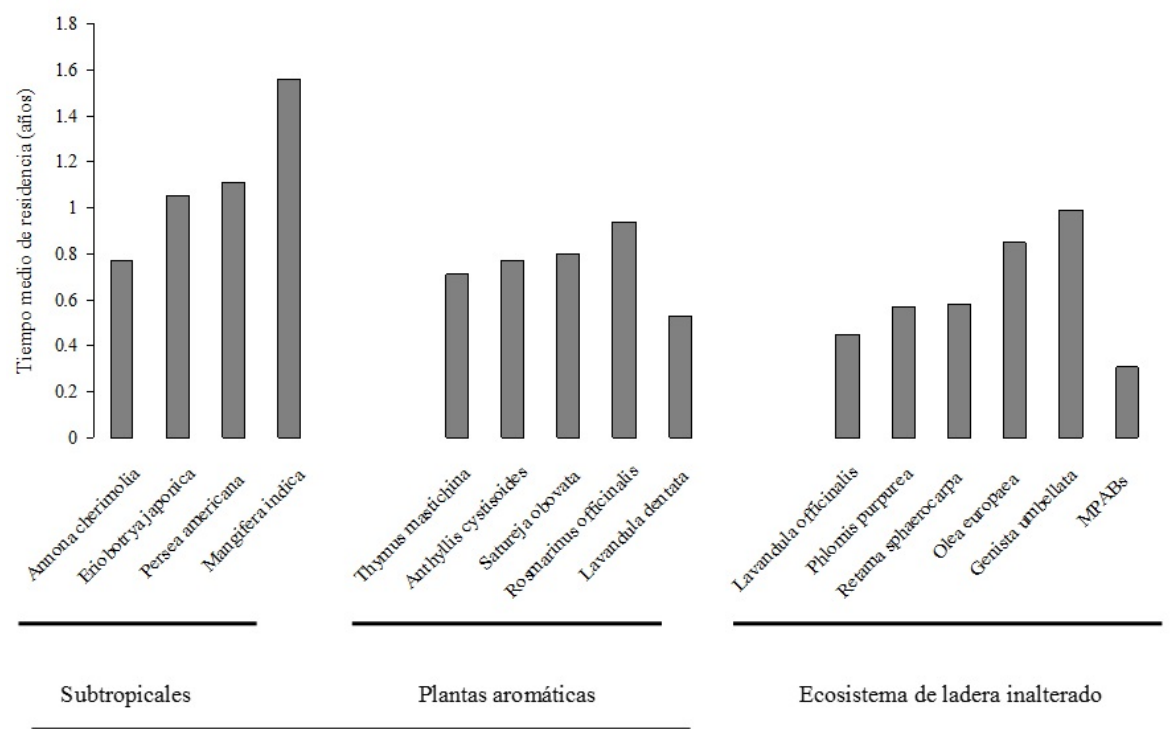

Figura 4. Tiempo medio de residencia para los escenarios estudiados.

Tabla 2. La liberación de $\mathrm{N}$ de cada especie en el ecosistema de ladera alterado (ELA)

\begin{tabular}{clllllllll}
\hline \multirow{2}{*}{ Etapa } & \multicolumn{4}{c}{ Cultivos subtropicales } & \multicolumn{5}{c}{ Plantas aromáticas y medicinales } \\
\cline { 2 - 12 } & Mango & Chirimoyo & Níspero & Aguacate & $\begin{array}{l}\text { Satureja } \\
\text { obovata }\end{array}$ & $\begin{array}{l}\text { Rosmarinus } \\
\text { officinalis }\end{array}$ & $\begin{array}{l}\text { Anthyllis } \\
\text { cytisoides }\end{array}$ & $\begin{array}{l}\text { Thymus } \\
\text { mastichina }\end{array}$ & $\begin{array}{l}\text { Lavandula } \\
\text { dentata }\end{array}$ \\
\hline 0 & 0 & 0 & 0 & 0 & 0 & 0 & 0 & 0 & 0 \\
1 & -0.34 & 0.42 & -1.08 & 0.22 & -0.99 & -0.68 & -0.77 & 0.53 & 0.13 \\
2 & -0.45 & 0.13 & -0.32 & 1.27 & -0.15 & -0.38 & -0.09 & 0.76 & 0.42 \\
3 & 0.10 & 0.55 & -0.15 & 1.36 & 0.60 & -0.32 & -0.16 & 0.90 & 0.24 \\
4 & 0.44 & 1.07 & 0.06 & 1.34 & 0.71 & -0.77 & -0.30 & 0.99 & 0.33 \\
Suma & -0.25 & 2.17 & -1.49 & 4.17 & 0.17 & -2.16 & -1.31 & 3.18 & 1.12 \\
\hline
\end{tabular}

Tabla 3. La liberación de $\mathrm{N}$ para cada especie en el ecosistema de ladera inalterado (ELI)

\begin{tabular}{cllllll}
\hline Etapa & $\begin{array}{l}\text { Genista } \\
\text { umbellata }\end{array}$ & $\begin{array}{l}\text { Lavandula } \\
\text { officinalis }\end{array}$ & $\begin{array}{l}\text { Olea } \\
\text { europaea }\end{array}$ & $\begin{array}{l}\text { Phlomis } \\
\text { purpurea }\end{array}$ & $\begin{array}{l}\text { Retama } \\
\text { sphaerocarpa }\end{array}$ & $\begin{array}{l}\text { Mezcla plantas } \\
\text { herbáceas }\end{array}$ \\
\hline 0 & 0 & 0 & 0 & 0 & 0 & 0 \\
1 & -0.16 & 1.47 & 0.24 & 1.63 & 0.33 & 0.32 \\
2 & -0.18 & 1.34 & 0.07 & 1.18 & 0.12 & - \\
3 & -0.22 & 1.12 & -0.02 & 0.42 & -0.48 & 0.41 \\
4 & -0.01 & 0.97 & -0.33 & 0.39 & -0.89 & 0.72 \\
5 & -0.15 & 1.14 & -0.55 & 0.76 & -0.75 & - \\
6 & -0.16 & 1.23 & -0.73 & 0.46 & -0.55 & 0.76 \\
Suma & -0.89 & 7.26 & -1.32 & 4.83 & -2.23 & 2.21 \\
Media ELI & & & 1.64 & & & \\
\hline
\end{tabular}

${ }^{1}$ Papaver rhoeas, Convolvulus sp., Reseda phyteuma, Anacyclus sp., Sinapis arvensis, Medicago sp., Poa annua, y Malva sylvestris.

\section{DISCUSIÓN}

El impacto ambiental que provoca el cambio de uso de suelo en un ecosistema natural a otro de tipo agrícola, es importante debido a la alteración de la flora y fauna (Figueroa y Sánchez 2008). Los residuos vegetales generados por la actividad agrícola contribuyen a la introducción de nuevos tipos de hojarasca, distinta a la autóctona de la zona, por lo que tienen una composición y característica distinta. Al respecto, Mubarak et al. (2008) repor$\tan$ que aproximadamente el $60 \%$ de la biomasa de 


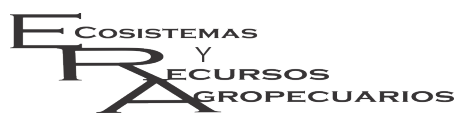

Rodríguez-Pleguezuelo et al.

Terreno y vegetación autóctona vs. agricultura

Ecosist. Recur. Agropec.

4(12):499-509,2017

mango (Mangifera indica L.) permanecía a los tres meses. Mientras que Vasconcelos et al. (2007) calcularon valores de k para Annona paludosa Aubl. de 1.13 años $^{-1}$, los cuales son menores a 1.65 años $^{-1}$ obtenidos en el presente estudio. La diferencia en el comportamiento de la hojarasca de frutales subtropicales se debe al tipo de hoja, ya que las de mango son duras y coriáceas, por lo que contribuyen de forma menos significativa al reciclado de nutrientes, mientras que las hojas de chirimoyo son más fáciles de degradar. En tanto que la hojarasca de aguacate y níspero se encuentran en un estado intermedio entre estos dos tipos de hojas.

Las PAM como agentes regeneradores y protectores de la erosión de los suelos en ambientes mediterráneos, han sido demostrada por Casermeiro (2002), Durán et al. (2006) y García et al. (2013). La descomposición de los restos de estas plantas proporciona beneficios en la mejora de la calidad de los suelos, aportando material orgánico. Al respecto Palm y Sánchez (1991) indica que este proceso en Thymus depende de factores, como la relación lignina: $\mathrm{N}$, la concentración de lignina o los polifenoles solubles. Los procesos de incorporación del material vegetal en los ecosistemas naturales son mucho más activos y efectivos. En este sentido, $G$. umbellata (L'Hér.) Dum. y O. europaea L. fueron las plantas con la mayor relación $\mathrm{C}: \mathrm{N}$ y las de mayor degradación. Similares resultados, obtuvieron Edmonds (1980), Moro y Domingo (2000). Por lo que la mezcla de plantas herbáceas anuales y bianuales alcanzaron las tasas más altas de descomposición. Esto se debe al tipo de plantas y al hecho de que exista una mezcla de plantas, ya que se ha demostrado que las pérdidas de masa en mezclas son mayores que cuando se trata de plantas aisladas (Gartner y Cardon 2004), lo que sugiere que ciertas interacciones entre plantas afectan la descomposición de la hojarasca.

No se encuentran estudios para la dinámica de $\mathrm{N}$ en la descomposición de hojarasca en diferentes ecosistemas. Pero recientemente Almeida et al. (2014) realizaron un estudio en mango sobre el ciclo bioquímico de nutrientes por medio de la evaluación de la concentración de $\mathrm{N}, \mathrm{P}, \mathrm{K}, \mathrm{Ca}, \mathrm{Mg}$ y $\mathrm{S}$ en las hojas nuevas, maduras, senescentes y hojarasca. De las hojas nuevas a las maduras, la concentración de $\mathrm{N}$ disminuyó de 13.9 a $5.7 \mathrm{~g} \mathrm{~kg}^{-1}$; sin embargo, desde la etapa de hojas senescentes a hojarasca incrementa, al igual que lo que ocurrió en el presente estudio. Cuando el cultivo de frutales sustituye a la vegetación autóctona, el ciclo natural de los nutrientes se ve alterado, con una movilización importante de N. Por lo que la alteración del ciclo del $\mathrm{N}$ en las laderas con terrazas y árboles frutales subtropicales podría compensarse con la instalación de cubiertas vegetales, para el control de la erosión, con altas tasas de reciclado de $\mathrm{N}$, como Th. mastichina L. con $3.18, L$. dentata L. con 1.12 , L. officinalis Chais con 7.26 o P. purpurea L. con 4.83. Se requiere avanzar en el estudio detallado del proceso del reciclado de nitrógeno por parte de las especies agrícolas introducidas, para comprender de manera exhaustiva el papel que estos residuos juegan en la mejora y aumento de la calidad de los suelos con cultivos en terrazas.

\section{CONCLUSIONES}

En los cultivos subtropicales estudiados, la hojarasca de chirimoyo y níspero se descomponen de forma más rápida, mientras que la hojarasca de mango y aguacate tiene un mayor periodo de degradación. Por lo que las hojas de chirimoyo pueden contribuir a un reciclado rápido del $\mathrm{N}$, mientras que el mango y el níspero sirven para la acumulación a largo plazo de N. Entre las PAM la hojarasca de L. dentata L. se descompone de forma rápida, produciendo una liberación de $\mathrm{N}$ desde el comienzo, mientras que $R$. officinalis L., $S$. obovata Lag y $A$. cystisoides $\mathrm{L}$. inmovilizaron el $\mathrm{N}$ los primeros cinco meses. Las plantas del ecosistema de ladera inalterado tuvieron mayores constantes de descomposición $(k)$, ya que, en el ecosistema de ladera alterado, las hojas de los cultivos subtropicales contribuyeron de forma lenta al reciclado de la biomasa. En ecosistema de ladera alterado, la implantación de cubiertas de PAM en los taludes de las terrazas, protegen al suelo frente de la erosión y favorecen el mantenimiento de las estructuras. Por 
lo que el cultivo de frutales subtropicales, puede ser sostenible desde el punto de vista del medio ambien- te, con la utilización de PAM en los taludes de las terrazas de cultivo.

\section{LITERATURA CITADA}

Aerts R (1997) Climate, leaf litter chemistry and leaf litter decomposition in terrestrial ecosystems: a triangular relationship. Oikos 79: 439-449.

Almeida CX, Pita JJL, Rozane DE, de Souza HA, Hemandes A, Natale W, et al. (2014) Nutrient cycling in mango trees. Ciências Agrárias 35: 259-266.

Barkman JJ (1989) A critical evaluation of the minimum area concepts. Vegetario 85: 89-104.

Casermeiro MA, de la Cruz C, Costa MT, Massanet JH, Molina MIH, Sánchez JA (2002) El papel de los tomillares (Thymus vulgaris L.) en la protección de la erosión del suelo. Anales de Biología 24: 81-87.

Castellanos BJ, León P (2011) Descomposición de hojarasca y liberación de nutrientes en plantaciones de Acacia mangium (Mimosaceae) establecidas en suelos degradados de Colombia. Revista Biología de Tropical 59: 113-128.

Cookson WR, Osman M, Marschner P, Abaye DA, Clarck I, Murphy DV, et al. (2007) Controls on soil nitrogen cycling and microbial community composition across land use and incubation temperature. Soil Biology and Biochemistry 39: 744-756.

Durán ZVH, Martínez RA, Aguilar RJ, Franco TD (2005) Impact of erosion in the taluses of subtropical orchard terraces. Agriculture, Ecosystems and Environment 107: 199-210.

Durán ZVH, Francia MJR, Rodríguez PCR, Martínez RA, Cárceles RB (2006) Soil-erosion and runoff prevention by plant covers in a mountainous area (SE Spain): implications for sustainable agriculture. Environmentalist 26: 309-319.

Durán ZVH, Rodríguez PCR (2009) Soil-erosion and runoff prevention by plant covers. In: Lichtfouse E, Navarrete M, Debaeke P (eds.). Sustainable agriculture. Springer Science+Business Media BV, The Netherlands. pp: 785-812.

Durán ZVH, García TIF, Francia MJR, Muriel RJL (2011) Soil erosion: causes, processes and effects. In: Fournier, A.J. (ed.). Soil Erosion: Causes, processes and effects. NovaScience Publishers, Hauppauge, New York, USA. pp: 1-34.

Durán ZVH, Rodríguez PCR, Francia MJR, Martín PFJ (2013) Land-use changes in a small watershed in the Mediterranean landscape (SE Spain): Environmental implications of a shift towards subtropical crops. Journal of Land Use Science 1: 47-58.

Durán ZVH, Rodríguez PCR, Cuadros TS, Francia MJR (2014) Impacto de la erosión y escorrentía en laderas de agroecosistemas de montaña mediterránea. Ecosistemas 23: 66-72.

Dutta RK, Agrawal M (2001) Litterfall, litter decomposition and nutrient release in five exotic plant species planted on coal mine spoils. Pedobiología 45: 298-312.

Edmonds RL (1980) Litter decomposition and nutrient release in Douglas-fir, red alder, western hemlock and Pacific silver fir ecosystems in western Washington. Canadian Journal of Forest Research 10: 327-337.

Elías F, Ruiz L (1977) Agroclimatología de España. Cuaderno del Instituto Nacional de Investigaciones Agrarias. Vol. 7, Ministerio de Agricultura. Madrid, España. 29p.

FAO (1998) FAO-SRIC-ISSS. World Reference Base for Soil Resources. Roma. Italy. 88p. 
Figueroa F, Sánchez CV (2008) Effectiveness of natural protected areas to prevent land use and land cover change in Mexico. Biodiversity and Conservation 17: 3223-3240.

García EP, Alonso BN, Marques MJ, Bienes R, González AF, Alegre J (2013) Use of Mediterranean legume shrubs to control soil erosion and runoff in central Spain. A large-plot assessment under natural rainfall conducted during the stages of shrub establishment and subsequent colonization. Catena 102: 3-12.

Gartner TB, Cardon ZG (2004) Decomposition dynamics in mixed-species leaf litter. Oikos 104: 230-246.

Heal OW, Anderson JM, Swift MJ (1997) Plant litter quality and decomposition: an historical overview. In: Cadisch G, Giller KE (eds.). Driven by nature: plant litter quality and decomposition. CAB International. Wallinford, Oxon, UK. pp: 3-30.

Kögel-Knabner I (2002) The macromolecular organic composition of plant and microbial residues as inputs to soil organic matter. Soil Biology and Biochemistry 34: 139-62.

Moriondo M, Good P, Durao R, Bindi M, Giannakopoulos C, Corte-Real J (2006) Potential impact of climate change on fire risk in the Mediterranean area. Climate Research 31: 85-95.

Moro MJ, Domingo F (2000) Litter decomposition in four woody species in a Mediterranean climate: weight loss, $\mathrm{N}$ and $\mathrm{P}$ dynamics. Annals of Botany 86: 1065-1071.

Mubarak AR, Elbashir AA, Elamin LA, Daldoum DMA, Steffens D, Benckiser G (2008) Decomposition and nutrient release from litter fall in the semi-arid tropics of Sudan. Communications in Soil Science and Plant Analysis 39: 2359-2377.

Olson JS (1963) Energy storage and the balance of producers and decomposers in ecological systems. Ecology 44: 322-331.

O'Neill EG, Johnson DW, Ledford J (2003) Acute seasonal drought does not permanently alter mass loss and nitrogen dynamics during decomposition of red maple litter. Global Change Biology 9: 117-23.

Palm CA, Sanchez PA (1991) Nitrogen release from the leaves of some tropical legumes as affected by their lignin and polyphenolic contents. Soil Biology and Biochemistry 23: 83-88.

Pandey RR, Sharma G, Tripathi SK, Singh AK (2007) Litterfall, litter decomposition and nutrient dynamics in a subtropical oak forest and managed plantation in northeastern India. Forest Ecology and Management 249: $96-104$

Rodriguez-Díaz J, Weatherhead EK, Knox JW, Camacho E (2007) Climate change impacts on irrigation water requirements in the Guadalquivir river basin in Spain. Regional Environmental Change 7: 149-159.

Rodríguez PCR, Durán ZVH, Francia MJR, Muriel FJL, Franco TD (2011) Monitoring the pollution risk and water use in orchard terraces with mango and cherimoya trees by drainage lysimeters. Irrigation and Drainage Systems 922: 83-90.

Romeo MN, Alberto MS, Sendros MD (2012) Seasonal changes in litter fall and its quality from three sub-tropical fruit trees at Nlespruit, South Africa. Agroforestry Systems 86: 61-71.

Santa-Regina I, Rapp M, Martin A, Gallardo JF (1997) Nutrient release dynamics in decomposing leaf litter in two Mediterranean deciduous oak species. Annals of Forest Science 54: 747-760.

Sariyildiz T, Anderson JM (2003) Interactions between litter quality, decomposition and soil fertility, a laboratory study. Soil Biology and Biochemistry 35: 391-399.

Sirulnik AG, Allen EB, Meixner T, Allen MF (2007) Impacts of anthropogenic N additions on nitrogen mineralization from plant litter in exotic annual grasslands. Soil Biology and Biochemistry 39: 24-32. 
Soil Survey Staff (1999) Soil Taxonomy. A Basic System of Soil Classification for Making and Interpreting Soil Surveys. Agric. Handbook No. 436. Natural Resources Conservation Service USDA. Washington, DC, USA. 869p.

Sun OJ, Campbell J, Law BE, Wolf V (2004) Dynamics of carbon storage in soils and detritus across chronosequences of different forest types in the Pacific Northwest USA. Global Change Biology 10: 1470-1481.

Tateno R, Tokuchi N, Yamanaka N, Du S, Otsuki K, Xue Z, et al. (2007) Comparison of litterfall production and leaf litter decomposition between an exotic black locust plantation and an indigenous oak forest near Yan'an on the Loess Plateau, China. Forest Ecology and Management 241: 84-90.

Taylor BR, Parkinson D, Parsons WFJ (1989) Nitrogen and lignin content as predictors of litter decay rates: a microcosm test. Ecology 70: 97-104.

Vasconcelos SS, Zarín DJ, da Rosa MB, Oliveira FA, Carvalho CJR (2007) Leaf decomposition in a dry season irrigation experiment in Eastern Amazonian forest regrowth. Biotropica 35: 593-600.

Waring EH, Schlesinger WH (1985) Forest Ecosystems, concepts and management. Academic Press, Orlando, Florida, USA. 338p.

Wang Z, Yin X, Li X (2015) Soil mesofauna effects on litter decomposition in the coniferous forest of the Changbai Mountains, China. Applied Soil Ecology 92: 64-71.

Zhang D, Hui D, Luo Y, Zhou G (2008) Rates of litter decomposition in terrestrial ecosystems: global patterns and controlling factors. Journal of Plant Ecology 1: 85-93.

Zhou G, Guan L, Wei X, Tang X, Liu S, Liu J, et al. (2008) Factors effecting leaf litter decomposition: an itersite decosmpiotion ecperimebt acroos China. Plant and Soil 311: 61-72. 
